\title{
DESEMPENHO DE MUDAS DE Pinus taed $a$ PRODUZIDAS EM RAIZ NUA E EM DOIS TIPOS DE RECIPIENTES, 24 MESES APÓS O PLANTIO
}

\author{
Adalberto Brito de Novaes ${ }^{1}$ \\ José Geraldo de A. Carneiro ${ }^{2}$ \\ Deborah Guerra Barroso ${ }^{3}$ \\ Paulo Sérgio Santos Leles ${ }^{4}$
}

\begin{abstract}
RESUMO
O presente estudo analisou a viabilidade do uso de uma nova metodologia de produção de mudas de Pinus taeda, cujo objetivo foi comparar a qualidade de mudas produzidas em blocos prensados, com mudas produzidas em tubetes e em raiz nua, com base nos parâmetros morfológicos. Empregou-se 5 tratamentos: a) Blocos prensados com $10 \mathrm{~cm}$ de altura; b) Blocos prensados com $7 \mathrm{~cm}$ de altura; c) Raiz nua; d) Tubetes (48 unidades/bandeja) e e) Tubetes (96 unidades/bandeja). Avaliou-se no viveiro os seguintes parâmetros: a) Altura da parte aérea (H); b) Diâmetro de colo (D); c) Relação H/D e d) Pesos de matéria fresca e seca das partes aérea, radicial e total. No campo foram avaliados a sobrevivência e o crescimento inicial. O desenvolvimento das mudas produzidas nos blocos prensados com $10 \mathrm{~cm}$ foi superior aos demais métodos utilizados neste trabalho. As médias mais baixas foram verificadas em mudas produzidas em tubetes.
\end{abstract}

Palavras-chave: qualidade de mudas, blocos prensados, viveiro, mudas

\section{PERFORMANCE Pinus taeda SEEDLINGS PRODUCED IN BARE ROOT AND TWO CONTEINER, AFTER 24 MONTHS OF THE PLANTATION}

\begin{abstract}
The present study has anlysed the viability of the use of a new methodology fase in the prodution of Pinus taeda seedlings. The main objective was to compare the seedlings quality when produced in pressed blocks, tubes $\left(60 \mathrm{~cm}^{3}\right)$ and bare root. The five treatment were: a) Pressed blocks $10 \mathrm{~cm}$ height; b) Pressed blocks $7 \mathrm{~cm}$ height; c) Bare roots; d) Tubes (48 units/tray) and e) Tubes (96 units/tray). Were evaluated in the nursery the following parameters: a) Stem height $(\mathrm{H})$; b) Root-collar diameter (D); c) H/D ratio and d) Weight fresh and dry weight matter (stem, root and total. In the field the survival and the initial growth. The performance of the seedlings produced in the $10 \mathrm{~cm}$ pressed blocks was higher than the other methods used in this experiment. The lowest averages were observed in seedlings produced in tubes for all morphological parameters evaluated.
\end{abstract}

Key-words: Seedlings quality, pressed blocks, nursery, forest seedlings

\section{INTRODUÇÃO}

A crescente expansão da área de plantios florestais no Brasil e as exigências do mercado forçam a busca de novas alternativas tecnológicas de mecanização para a maioria das operações que envolvem essas atividades. Para atingir as metas de um programa de reflorestamento e alcançar povoamentos mais produtivos, principalmente, deve-se escolher criteriosamente a espécie e o melhor sistema de produção de mudas. Uma muda considerada de alto padrão de qualidade deve suportar as adversidades do meio, apresentar altos porcentuais de sobrevivência no campo, bom crescimento para que se possa reduzir a

\footnotetext{
${ }^{1}$ Adalberto Brito de Novaes - Doutor - Dep. de Fitotecnia da Universidade Estadual do Sudoeste da Bahia. Estrada do Bem Querer, km 4, Vitória da Conquista-BA, 45.100-000. adalbert@uesb.br

2 José Geraldo de A. Carneiro - PhD - CCTA da Universidade Estadual do Norte Fluminense, Av.Alberto Lamego, 2.000, Campos dos Goytacazes-RJ, 28015-620. carneiro@uenf.br

${ }^{3}$ Deborah Guerra Barroso - Doutora - CCTA da Universidade Estadual do Norte Fluminense, Av. Alberto Lamego, 2.000, Campos dos Goytacazes-RJ, 28015-620. deborah@uenf.br

${ }^{4}$ Paulo Sérgio Santos Leles - Doutor - Dep. de Silvicultura da Universidade Federal Rural do Rio de JaneiroUFRJ, BR 465, km 07, Seropédica. pleles@ufrrj.br
} 
frequiência dos tratos culturais do povoamento recém implantado e produzir árvores com volume e qualidades desejáveis. Entretanto, o aperfeiçoamento das técnicas de produção de mudas não conseguiu acompanhar o progresso alcançado nas demais etapas das atividades de reflorestamento (GOMES et al. 1977). Segundo PARVIAINEN \& ANTOLA (1986) as exigências econômicas têm forçado o máximo de mecanização do processo de produção de mudas em recipientes. No Brasil, muitos problemas ainda existem, principalmente em se tratando de mudas produzidas em recipientes que não causem danos a uma adequada formação do sistema radicial, pois segundo CARNEIRO (1995), os recipientes devem apresentar formas e volumes que evitem o crescimento de raízes mal formadas.

Muitos são os autores que se posicionaram favoravelmente ao sistema de produção de mudas em recipientes, comparativamente ao sistema de raiz nua. As dimensões dos recipientes e o volume destinado ao enraizamento influenciam na disponibilidade de suprimento de nutrientes e água. As mudas terão o seu desenvolvimento afetado, caso esta disponibilidade seja muito limitada ou presente em quantidade considerada excessiva (BÖHM, 1979). É de grande importância o estudo das dimensões adequadas dos recipientes. Volume acima do recomendado provoca gastos desnecessários, como o aumento da área do viveiro e dos custos de transporte e, ainda, de manutenção e distribuição das mudas no campo (GONZALEZ ROQUE et al. 1988).

Segundo SCHMIDT-VOGT (1984), deve-se priorizar a produção de mudas sem deformações radiciais, com o propósito de alcançar maiores incrementos médios anuais. Ressaltou, ainda, que a produção de mudas em recipientes deve proporcionar a formação de fortes sistemas radiciais, com o mínimo de deformações. Também CARNEIRO (1987) recomendou que os recipientes não devem provocar dobras e crescimento das raízes em espiral.

Para SJOBERG (1974), HULTÉN (1974) \& TINUS \& McDONALD (1979), os recipientes devem respeitar as características biológicas das espécies, não provocando danos à conformação natural das raízes, para que sejam alcançados expressivos índices de sobrevivência e desenvolvimento após o plantio. A restrição radicial imposta por recipientes, segundo CARNEIRO (1995), pode diminuir a resistência à desidratação, com prejuízo para sua condição hídrica. A orientação horizontal das raízes laterais foi ressaltada, tendo em vista que alguns problemas após o plantio podem estar relacionados com os tipos de recipientes. $\mathrm{O}$ desempenho de mudas de Pinus taeda, originárias da semeadura direta no campo e o da produção em tubetes, foi estudado por MATTEI (1993). O autor verificou que todas as plantas originárias da semeadura apresentaram raízes laterais distribuídas na forma horizontal, o tubete mostrou-se inadequado para a produção de mudas de Pinus taeda, induzindo à deformação das raízes laterais, podendo trazer conseqüências negativas para o crescimento futuro das mudas no campo. Já as mudas originadas da semeadura direta, apresentaram melhor desempenho quando comparadas às produzidas em tubetes.

ALM \& SCHANTZ-HANSEN (1974) comentaram que recipientes pequenos, tipo tubetes, são inadequados, pois restringem o crescimento do sistema radicial não tendo proporcionado quantidade adequada de meio para o enraizamento de mudas de espécies do gênero Pinus.

As dimensões dos tubetes para $60 \mathrm{~cm}^{3}$, em decorrência da restrição radicial, segundo BARROS et al. (1978), podem causar uma reduzida taxa de desenvolvimento no viveiro. Contudo, esses autores, trabalhando com Eucalyptus grandis, verificaram a recuperação do crescimento em altura após o plantio. Segundo CARNEIRO (1995), espécies do gênero Eucalyptus são mais tolerantes à restrição radicial que as de Pinus. Entretanto, HENRIQUES et al. (1987) não encontraram desenvolvimento satisfatório para mudas de Eucalyptus cloeziana \& E. pyrocarpa.

PARVIAINEN (1984) recomendou o uso de blocos prensados de turfa $(60 \times 40 \mathrm{~cm})$, o qual possibilita o desenvolvimento do sistema radicial em sua posição natural, livre, sem confinamento ou direcionamento das raízes, usualmente provocados por paredes de recipientes. De acordo com PARVIAINEN (1990), este sistema possibilita às mudas um 
favorável desenvolvimento após o plantio, apresentando um sistema radicial praticamente isento de deformações. Segundo PARVIAINEN \& TERVO (1989), cada bloco contém 96 pontos de semeadura, espaçados de $5 \mathrm{~cm}$. Por ocasião da expedição das mudas para o plantio, os blocos são serrados, mecanicamente, em sentidos transversais para a individualização dos torrões que contém as mudas. As serras promovem a poda das raízes laterais e possibilita o desenvolvimento radicial com o decorrente aumento do número de extremidades de raízes. As evidências apresentadas demonstram, a necessidade de novas tecnologias de produção de mudas em recipientes, visando atingir os melhores padrões de qualidade desejados, otimizando assim, o sistema de produção de mudas de espécies florestais. Neste sentido, o objetivo desse trabalho foi testar uma nova metodologia de produção de mudas de Pinus taeda L., comparando a eficiência do sistema de blocos prensados com sistemas convencionais, visando a produção de mudas que apresentassem características de maiores taxas de sobrevivência e crescimento inicial após o plantio.

\section{MATERIAL E MÉTODOS}

As sementes de Pinus taeda foram coletadas em árvores geneticamente melhoradas (PS). A região em que foi conduzido o experimento bem como, a coleta das sementes, situa-se à latitude de $26^{\circ} 07^{\prime} \mathrm{S}$ e longitude $50^{\circ} 19^{\prime} \mathrm{W}$. A altitude é de $775 \mathrm{~m}$ sobre o nível do mar. O clima da região, segundo a classificação de Köppen, é do tipo Cfb, sendo a temperatura média do mês mais quente, inferior a $22^{\circ} \mathrm{C}$ e a do mês mais frio, superior a $10^{\circ} \mathrm{C}$, ocorrendo mais de 10 geadas por ano. A precipitação média anual é de $1.434 \mathrm{~mm}$.

\section{RECIPIENTES UTILIZADOS PRODUÇÃO DAS MUDAS}

NA

Tubetes

O modelo de tubete foi o cônico de secção circular contendo quatro frisos internos longitudinais e eqüidistantes com as seguintes dimensões: $12,5 \mathrm{~cm}$ de altura e $3 \mathrm{~cm}$ de diâmetro na parte interna superior, apresentando $60 \quad \mathrm{~cm}^{3}$ de capacidade volumétrica de substrato.

\section{Blocos prensados}

Os blocos prensados foram produzidos pela empresa finlandesa VAPO OY. Com espessuras de 2 e $3 \mathrm{~cm}$ e capacidade de expansão de 7 e $10 \mathrm{~cm}$, respectivamente, quando submetidos às regas normais. Cada bloco continha 96 orifícios de aproximadamente $2 \mathrm{~mm}$ de profundidade, espaçados de $5 \times 5 \mathrm{~cm}$ nos quais foram colocadas as sementes. Os blocos foram postos em caixas de material plástico rígido com dimensões de $60 \times 40 \mathrm{~cm}$, com alturas iguais às espessuras dos blocos expandidos. Quando as mudas atingiram a altura ideal para $o$ plantio, os blocos foram serrados nos sentidos transversal e longitudinal, individualizando-se as mudas em torrões.

\section{TRATAMENTOS}

Foram considerados cinco tratamentos, envolvendo três sistemas de produção de mudas: 1) Bloco 10 (Sistema de blocos prensados com $10 \mathrm{~cm}$ de altura após a expansão); 2) Bloco 7 (Sistema de blocos prensados com $7 \mathrm{~cm}$ de altura após a expansão); 3) Raiz nua; 4) Tubete com menor densidade (48 mudas/bandeja - D48) e 5) Tubete com maior densidade (96 mudas/bandeja - D96). No tratamento 4, as mudas foram distribuídas uniformemente em cada bandeja, deixando-se livre uma célula correspondente à posição de um tubete entre elas. No tratamento 5, os tubetes foram distribuídos em todas as células. Cada parcela de $1 \mathrm{~m}^{2}$, correspondente ao tratamento em raiz nua, apresentou uma densidade média de 240 mudas.

\section{PRODUÇÃO DAS MUDAS}

O substrato para enchimento dos tubetes foi uma mistura de matéria orgânica humificada à base de turfa de $\mathrm{pH}=5,9$ e com os seguintes níveis de fertilidade (\%): $\mathrm{N}$ (total) $=0,90 ; \mathrm{P}=2,66 ; \mathrm{K}=0,32$; Matéria Orgânica $=$ 36,0 .

A semeadura foi feita manualmente, colocando-se três sementes em cada tubete e orifício de cada bloco prensado. Como cobertura morta foi usada uma camada de acículas secas picadas de pinus, com uma 
espessura de $1 \mathrm{~cm}$. As regas foram efetuadas diariamente durante a formação das mudas.

As mudas em raiz nua foram escolhidas de forma aleatória, em seis diferentes canteiros de um viveiro comercial. O substrato utilizado correspondeu ao próprio solo do viveiro, preparado através das operações de aração, gradagem, emprego de enxada rotativa, calagem e aplicação de adubação mineral e orgânica.

\section{PARÂMETROS MORFOLÓGICOS}

As avaliações aos seis meses de idade foram feitas em amostras de 20 mudas por parcela em cada tratamento, através dos seguintes parâmetros: a) Altura da parte aérea (H); b) Diâmetro de colo (D); c) Relação H/D; d) Peso de matéria fresca da parte aérea e) Peso de matéria fresca do sistema radicial; f) Peso de matéria fresca total; g) Peso de matéria seca da parte aérea; h) Peso de matéria seca do sistema radicial; i) Peso de matéria seca total. Para a determinação desses parâmetros, efetuou-se uma lavagem no sistema radicial das mudas, visando a separação de todos os resíduos de substrato aderidos às raízes. Posteriormente, as mudas foram postas sobre folhas de jornal por um período de 24 horas, a partir do qual, foram feitas as medições de altura da parte aérea, diâmetro de colo e as respectivas pesagens de matéria fresca. Foram preparadas, para a secagem das mudas, duas embalagens de papel, uma contendo a parte aérea e outra o sistema radicial que, após etiquetadas, foram colocadas em estufa previamente aquecida a $75^{\circ} \mathrm{C}$, conforme orientações de SCHUURMAN \& GOEDEWAAGEN (1971), citados por BÖHM (1979).

\section{PLANTIO NO CAMPO}

As mudas produzidas em blocos prensados foram individualizadas, através do corte dos blocos com uma serra manual, 10 dias antes do plantio, período que permitiu o início da regeneração das raízes podadas, ainda no viveiro. $O$ espaçamento no campo foi de 3,0 $\mathrm{x} \quad 2,5 \mathrm{~m}$, sendo $\mathrm{o}$ plantio efetuado manualmente. A determinação da porcentagem de sobrevivência foi efetuada a cada 30 dias, durante cinco meses. A cada seis meses (durante dois anos) foi efetuada uma avaliação da altura da parte aérea e do diâmetro ao nível do solo.

\section{ANÁLISE ESTATÍSTICA}

$\mathrm{O}$ delineamento experimental usado no viveiro foi o de inteiramente ao acaso, com 5 tratamentos e 6 repetições. Nos tratamentos 1 , 2, e 5, cada parcela foi composta com 384 mudas distribuídas em quatro bandejas e no tratamento 4 , manteve-se o mesmo número de mudas porém, distribuídas em oito bandejas, dado a menor densidade.

No campo, o delineamento experimental usado foi o de blocos ao acaso com 6 repetições e parcelas constituídas de 30 mudas. As médias dos tratamentos foram comparadas pelo teste de Duncan a 5\% de probabilidade.

\section{RESULTADOS E DISCUSSÃO}

\section{AVALIAÇÃO DOS PARÂMETROS MORFOLÓGICOS}

\section{Altura da parte aérea, diâmetro de colo e relação H/D}

Os resultados médios de altura da parte aérea $(\mathrm{H})$, diâmetro de colo $(\mathrm{D})$ e relação H/D são apresentados na Tabela 1 . O sistema de blocos prensados com $10 \mathrm{~cm}$ produziu mudas maiores, embora não tenha havido diferença estatística em relação à média obtida pelas mudas produzidas no sistema de raiz nua. BACON et al. (1977), trabalhando com mudas de um ano de Pinus elliottii, recomendaram que a altura média das mudas deva situar-se entre 15 e $40 \mathrm{~cm}$. Os blocos prensados com 7 $\mathrm{cm}$ produziram mudas de menores alturas em relação àquelas produzidas pelos blocos prensados com $10 \mathrm{~cm}$ e raiz nua, porém, superiores às produzidas em tubetes. Estes resultados são similares aos verificados por CARNEIRO (1995), que encontrou para mudas de Pinus taeda, produzidas em blocos prensados com $7 \mathrm{~cm}$, maiores médias de altura da parte aérea, em relação às mudas produzidas em tubetes.

Quanto ao diâmetro de colo, a maior média $(3,45 \mathrm{~mm})$ também foi observada nas mudas produzidas no sistema de blocos prensados com $10 \mathrm{~cm}$, seguido das mudas produzidas em raiz nua. As menores médias foram verificadas para mudas produzidas em 
tubetes. As mudas produzidas em blocos prensados apresentaram desenvolvimento em diâmetro de colo bastante superior em relação àquelas produzidas em tubetes. CARNEIRO \& PARVIAINEN (1988) também constataram que mudas de Pinus taeda, originadas do sistema de blocos prensados com $7 \mathrm{~cm}$ apresentaram maior diâmetro médio, quando comparadas às mudas produzidas em tubetes. Trabalhando com mudas dessa espécie, CARNEIRO (1995) também constatou maior diâmetro médio de mudas produzidas em blocos prensados com $7 \mathrm{~cm}$, enquanto os tubetes produziram mudas de menores valores. SOUTH et al. (1993) constataram que o crescimento no campo de mudas de Pinus radiata foi maior quando estas apresentaram maiores diâmetros de colo, independentemente de sítios e tratamentos.
As maiores médias para a relação H/D foram obtidas em mudas produzidas em raiz nua e blocos prensados com $10 \mathrm{~cm}$, valores que revelam, segundo CARNEIRO (1995), que as mudas deveriam apresentar diâmetros maiores para maior equilíbrio do desenvolvimento da parte aérea. Também, SCHMIDT-VOGT \& GÜRTH (1969) constataram que para maior crescimento em altura, as mudas devem apresentar compatível desenvolvimento em diâmetro. As mudas dos demais tratamentos, particularmente as dos blocos prensados com $7 \mathrm{~cm}$ e tubete D96, apresentaram médias menores desta relação, embora tenham apresentado valores de diâmetro muito pequenos, indicativos de mudas de qualidade inferior.

Tabela 1 - Médias de altura da parte aérea, diâmetro de colo e relação H/D de mudas de Pinus taeda, seis meses após a semeadura.

\begin{tabular}{lccc}
\hline \hline Tratamento & Altura da parte aérea $(\mathrm{H})(\mathrm{cm})$ & Diâmetro de colo $(\mathrm{D})(\mathrm{mm})$ & $\begin{array}{c}\text { RELAÇÃ } \\
\text { H/D }\end{array}$ \\
\hline Bloco 10 & & $3,45 \mathrm{a}$ & $9,52 \mathrm{~b}$ \\
Raiz Nua & $32,56 \mathrm{a}$ & $3,05 \mathrm{~b}$ & $10,00 \mathrm{a}$ \\
Bloco 7 & $30,06 \mathrm{a}$ & $2,60 \mathrm{c}$ & $8,55 \mathrm{c}$ \\
Tubete D96 & $22,07 \mathrm{~b}$ & $1,66 \mathrm{~d}$ & $7,25 \mathrm{~d}$ \\
Tubete D48 & $11,51 \mathrm{c}$ & $1,61 \mathrm{~d}$ & $6,11 \mathrm{e}$ \\
\hline
\end{tabular}

Médias seguidas pela mesma letra não diferem entre si, ao nível de 5\% de probabilidade, pelo teste de Duncan.

\section{Peso de matéria fresca}

Verifica-se, na Tabela 2, que as médias das mudas produzidas em blocos prensados com $10 \mathrm{~cm}$ apresentaram o maior peso de matéria fresca para os três parâmetros pesquisados, seguindo-se as mudas de raiz nua e as produzidas em blocos prensados com 7 cm. CARNEIRO \& BRITO (1992), também encontraram maiores valores de peso de matéria fresca para os mesmos parâmetros pesquisados em mudas produzidas em raiz nua em relação às produzidas em blocos prensados com $7 \mathrm{~cm}$. SCHMIDT-VOGT (1966), constatou que mudas da mesma espécie com maior peso de raízes apresentam maiores chances de sobrevivência no campo. As menores médias de peso de matéria fresca para os três parâmetros pesquisados foram observadas nas mudas produzidas em tubetes.

Tabela 2 - Pesos de matéria fresca das partes aérea, radicial e total de mudas de Pinus taeda, seis meses após a semeadura.

\begin{tabular}{|c|c|c|c|}
\hline \multirow[t]{2}{*}{ Tratamento } & \multicolumn{3}{|c|}{ Peso de matéria fresca } \\
\hline & Parte aérea $(\mathrm{g})$ & Raiz $(\mathrm{g})$ & Total $(\mathrm{g})$ \\
\hline Bloco 10 & $5,31 \mathrm{a}$ & $0,58 \mathrm{a}$ & $5,89 \mathrm{a}$ \\
\hline Raiz Nua & $4,20 \mathrm{~b}$ & $0,43 \mathrm{~b}$ & $4,62 \mathrm{~b}$ \\
\hline Bloco 7 & $2,41 \mathrm{c}$ & $0,32 \mathrm{c}$ & $2,73 \mathrm{c}$ \\
\hline Tubete D48 & $0,56 \mathrm{~d}$ & $0,28 \mathrm{~cd}$ & $0,84 \mathrm{~d}$ \\
\hline
\end{tabular}


Tubete D96

$0,54 \mathrm{~d}$

$0,23 \mathrm{~d}$

$0,77 \mathrm{~d}$

Médias seguidas pela mesma letra não diferem entre si, ao nível de 5\% de probabilidade, pelo teste de Duncan

\section{Peso de matéria seca}

Verifica-se, na Tabela 3, que não não houve diferença estatística entre as médias das mudas produzidas em raiz nua e blocos prensados com $10 \mathrm{~cm}$, para os três parâmetros em análise. Contudo, ressalta-se que parte das raízes das mudas produzidas nos blocos prensados foi eliminada através da poda do sistema radicial. As raízes das mudas produzidas no sistema de raiz nua também foram submetidas à uma poda, porém, menos drástica. As médias de peso de matéria seca radicial das mudas produzidas nos sistemas de blocos prensados com $7 \mathrm{~cm}$, tubetes de maior e menor densidades não apresentaram diferença significativa. Também nos blocos com $7 \mathrm{~cm}$, as raízes foram podadas, em contraposição com as dos tubetes, que não sofreram poda. Este efeito fica evidenciado na análise de peso de matéria seca das partes aérea e total dos blocos prensados com $7 \mathrm{~cm}$, cujas médias, equivalentes à da raiz nua, diferiram significativamente dos tratamentos constituídos pelos tubetes. CARNEIRO \& BRITO (1992) também encontraram maiores valores de peso de matéria seca para os mesmos parâmetros pesquisados em mudas produzidas em raiz nua em relação às produzidas em blocos prensados com $7 \mathrm{~cm}$. O peso de matéria seca da parte aérea é uma boa indicação da capacidade de resistência das mudas (SCHMIDT-VOGT, 1966). Esta constatação foi confirmada também por CARNEIRO (1976).

Tabela 3 - Peso de matéria seca das partes aérea e radicial e peso total de mudas de Pinus taeda, seis meses após a semeadura.

\begin{tabular}{lccc}
\hline \hline Tratamento & $\begin{array}{c}\text { Peso de matéria seca } \\
\text { raiz }(\mathrm{g})\end{array}$ & $\begin{array}{c}\text { Peso de matéria seca aéreo } \\
(\mathrm{g})\end{array}$ & $\begin{array}{c}\text { Peso de matéria } \\
\text { seca total }(\mathrm{g})\end{array}$ \\
\hline Bloco 10 & $0,23 \mathrm{a}$ & $1,87 \mathrm{a}$ & $2,10 \mathrm{a}$ \\
Raiz Nua & $0,25 \mathrm{a}$ & $1,65 \mathrm{ab}$ & $1,88 \mathrm{ab}$ \\
Bloco 7 & $0,15 \mathrm{~b}$ & $1,04 \mathrm{~b}$ & $1,20 \mathrm{~b}$ \\
Tubete D96 & $0,15 \mathrm{~b}$ & $0,25 \mathrm{c}$ & $0,41 \mathrm{c}$ \\
Tubete D48 & $0,15 \mathrm{~b}$ & $0,24 \mathrm{c}$ & $0,40 \mathrm{c}$ \\
\hline
\end{tabular}

Médias seguidas pela mesma letra não diferem e entre si, ao nível de 5\% de probabilidade, pelo teste de Duncan.

\section{DESEMPENHO DAS MUDAS NO CAMPO}

\section{Sobrevivência das mudas}

Os maiores índices de sobrevivência (Tabela 4) couberam às mudas produzidas no sistema de blocos prensados com 10 e $7 \mathrm{~cm}$ $(98,34 \%$ e $\quad 97,94$ respectivamente).
CARNEIRO (1995) também constatou para mudas de Pinus taeda, maiores médias de sobrevivência verificadas para as mudas produzidas no sistema de blocos prensados com $7 \mathrm{~cm}$. As mudas produzidas no sistema de raiz nua alcançaram menor taxa de sobrevivência $(88,89)$.

Tabela 4 - Sobrevivência de mudas de Pinus taeda, cinco meses após o plantio.

\begin{tabular}{cc}
\hline \hline TRATAMENTO & Sobrevivência (\%) \\
\hline Bloco10 & 98,34 \\
Bloco 7 & 97,94 \\
Tubete D96 & 96,67 \\
Tubete D48 & 95,56 \\
Raiz nua & 88,89 \\
\hline
\end{tabular}




\section{Crescimento inicial em altura}

Verifica-se (Tabela 5) que as mudas produzidas no sistema de blocos prensados com $10 \mathrm{~cm}$ apresentaram a maior média de altura 24 meses após o plantio. A segunda maior média coube às mudas dos blocos prensados com $7 \mathrm{~cm}$. CARNEIRO (1995), pesquisando mudas de Pinus taeda em blocos prensados com $7 \mathrm{~cm}$, raiz nua e tubetes, observou que as maiores médias de altura, em sítios arenoso e argiloso, foram verificadas para as mudas produzidas pelo primeiro e segundo métodos, respectivamente. RICHTER (1971), pesquisando mudas de Pseudotsuga menziesii, verificou que as mudas mais altas apresentaram maiores taxas de crescimento no campo. As mudas produzidas em tubetes apresentaram as médias mais baixas

Tabela 5 - Altura das árvores de Pinus taeda, 24 meses após o plantio.

\begin{tabular}{cc}
\hline \hline Tratamento & Altura $(\mathrm{cm})$ \\
\hline Bloco 10 & $275,50 \mathrm{a}$ \\
Bloco 7 & $251,00 \mathrm{~b}$ \\
Raiz Nua & $242,67 \mathrm{bc}$ \\
Tubete D96 & $236,67 \mathrm{bc}$ \\
Tubete D48 & $226,17 \mathrm{c}$ \\
\hline
\end{tabular}

Médias seguidas pela mesma letra não diferem entre si, ao nível de 5\% de probabilidade, pelo teste de Duncan.

Os resultados obtidos, 24 meses após o plantio, demonstraram a superioridade das mudas produzidas por meio do sistema de blocos prensados com $10 \mathrm{~cm}$, que apresentaram maior ritmo de crescimento inicial em altura. Neste período, foram constatados ritmos de crescimento similares para as mudas produzidas em blocos prensados com $7 \mathrm{~cm}$ e em raiz nua, com uma ligeira tendência de superioridade do primeiro no final deste período. Os resultados de crescimento em altura alcançados pelas mudas produzidas em blocos prensados com $10 \mathrm{~cm}$, sugerem a possibilidade de uma provável redução nos custos de manutenção, em relação ao plantio efetuado com mudas em raiz nua.

A diferença da espessura dos blocos prensados permitiu a produção de mudas de diferentes alturas, prevalecendo com maior desenvolvimento inicial no campo, as mudas com maiores dimensões oriundas de blocos mais altos. BARNETT (1983), trabalhando com mudas de Pinus taeda, $P$. elliottii, $P$. echinata e $P$. palustris produzidas em recipientes, verificou que o desempenho no campo foi maior, à medida em que as dimensões das mudas, no ato do plantio, também foram maiores.

O ritmo mais lento e os baixos valores de crescimento em altura no campo, verificados em mudas produzidas em tubetes, mostram que este recipiente não é recomendável para espécies de Pinus, pois causa restrição ao sistema radicial motivo pelo qual suas médias foram muito baixas, até pelo menos 24 meses após o plantio. Chegaram à mesma constatação, ALM \& SCHANTZHANSEN (1974), MATTEI (1993) \& CARNEIRO (1995), após resultados similares da avaliação dos parâmetros morfológicos e desempenho no campo verificados em mudas de Pinus taeda.

\section{Crescimento inicial em diâmetro ao nível do solo}

Verificou-se que as mudas produzidas no sistema de blocos prensados com $10 \mathrm{~cm}$ apresentaram a maior média de diâmetro, destacando-se dos demais (Tabela 6). As mudas produzidas em raiz nua e blocos prensados com $7 \mathrm{~cm}$ também apresentaram, no campo, entre estes tratamentos, ritmos de crescimento similares. Todavia, apesar das mudas em raiz nua terem apresentado valores superiores na fase de viveiro, foi o sistema de blocos prensados com $7 \mathrm{~cm}$ que obteve o melhor crescimento no campo. Os resultados confirmaram que o melhor desempenho, neste período, coube às mudas produzidas em blocos prensados, destacando-se aquelas produzidas em blocos prensados com $10 \mathrm{~cm}$, mostrando a vantagem desse sistema de produção. CARNEIRO (1995) também encontrou as maiores médias de diâmetro, ao nível do solo, em mudas de Pinus taeda produzidas em blocos prensados com $7 \mathrm{~cm}$, em comparação 
com as mudas produzidas em raiz nua e tubetes. As mudas produzidas no sistema de tubetes apresentaram ritmo de crescimento mais lento. Seus baixos valores de diâmetro ao nível do solo, demonstraram mais uma vez a desvantagem desse recipiente para a produção de mudas de espécies de Pinus. Esta constatação também foi confirmada por ALM \& SCHANTZ-HANSEN (1974), MATTEI (1993) e CARNEIRO (1995).

Tabela 6 - Diâmetro ao nível do solo de árvores de Pinus taeda, 24 meses após o plantio.

\begin{tabular}{cc}
\hline \hline Tratamento & Diâmetro (mm) \\
\hline Bloco 10 & $61,09 \mathrm{a}$ \\
Bloco 7 & $52,86 \mathrm{~b}$ \\
Raiz Nua & $52,75 \mathrm{~b}$ \\
Tubete D96 & $47,01 \mathrm{c}$ \\
Tubete D48 & $45,54 \mathrm{c}$ \\
\hline
\end{tabular}

Médias seguidas pela mesma letra não diferem entre si, ao nível de 5\% de probabilidade, pelo teste de Duncan.

\section{CONCLUSÕES}

Nas condições em que foi realizado os experimentos, conclui-se que:

1. O sistema de blocos prensados com $10 \mathrm{~cm}$, em todas as fases de avaliação dos parâmetros de viveiro e desempenho no campo até 24 meses após o plantio, foi superior aos demais métodos utilizados neste trabalho, demonstrando assim, a sua eficiência na produção de mudas de Pinus taeda.

2. As médias mais baixas, para todos os parâmetros morfológicos avaliados e desempenho no campo até 24 meses após o plantio, foram verificadas em mudas produzidas em tubetes.

\section{BIBLIOGRAFIA CITADA}

ALM, A. A.; SCHANTZ-HANSEN, R. Tubeling research plantings Minnesota. In: NORTH AMERICAN

CONTAINERIZED FOREST TREE SEEDLING SYMPOSIUM, 1974, Denver; Colorado). Proceedings...

(Washington, D. C.: U. S.). Government Printing Office, 1974. p.384-387. (Great Plains Agric. Council Publication, 68).

BACON, G. J.; HAWKINS, P.J.; JERMYN, D. Morphological granding studies with 1-0 slash pine seedlings. Aus. For., Queensland, v. 40, p. 293-303, 1977.

BARNETT J. P. Relating seedling morphology of container- grown southern pines to field success. Separata de: CONVENTION OF THE SOCIETY OF AMERICAN
FORESTERS (1983: Portland).

Proceedings..,. New Orleans: USDA. For. Serv. Southern Forest Experiments Station, 1983. p. 405-407.

BARROS, N. F. et al. Efeitos de recipientes na sobrevivência e no crescimento de mudas de Eucalyptus grandis S. Hill ex Maiden, no viveiro e no campo. Revista Árvore, Viçosa, v.2, n.2 p. 141-151. 1978.

BÖHM, W. Methods of studying root systems. Berlin: Springer- Verlag, 1979. 188 p.

CARNEIRO, J. G. de A. Determinação do padrão de qualidade de mudas de Pinus taeda, L. para plantio definitivo. Curitiba: UFPR, 1976. 70 f. Dissertação Mestrado

CARNEIRO, J. G. de A. Influência de recipientes e de estações de semeadura sobre o comportamento do sistema radicular e dos parâmetros, morfológicos de mudas de Pinus taeda e Pinus elliottii. Curitiba: UFPR 1987. 81f.

CARNEIRO, J. G. de A. Produção e controle de qualidade de mudas florestais. Curitiba: UFPR/ FUPEF, Campos: UENF, 1995. 451p.

CARNEIRO, J. G. de A.; BRITO, M. A. R. Nova metodologia para produção mecanizada de mudas de Pinus taeda L. em recipientes com raízes laterais podadas. Floresta, Curitiba, v. 22, n.1/2, p.63-77, 1992.

CARNEIRO, J. G. de A. ; PARVIAINEN, J. $\mathrm{V}$. Comparison of production methods for containerized pine (Pinus elliottii) seedlings in Southern Brazil. 
Metsantutkimuslaitoksen Tiedonantoja, Joensuu, Finlândia, n.302, p. 6-24, 1988.

GOMES, J. M. et al. Efeitos de recipientes e substratos na produção de mudas de Eucalyptus grandis W. Hill ex Maiden. Revista Árvore, Viçosa, v.1, n.2, p.167-172, 1977.

GONZALEZ ROQUE, A. et al. Estudio sobre el comportamiento en vivero de Pinus caribaea var. caribaea cultivado en envases de polietileno de 12 dimensiones diferentes. Revista Forestal Baracoa, Havana, v.18, n.1, p.39-51, 1988.

HENRIQUES, E. P. et al. Produção de mudas na ACESITA ENERGÉTICA S.A. Série Técnica IPEF, Piracicaba, v.4, n.13, p.1317, 1987.

HULTÉN, H. Containerization in Scandinavia. In: NORTH AMERICAN CONTAINERIZED FOREST TREE SEEDLINGS SYMPOSIUM (1974.: Denver, Col.), Proceedings... (Washington, D. C.): U.S. Government Printing office, 1974, p.20-28. (Great Plains Agric. Council Publication, 68).

MATTEI, V. L. Comparação entre semeadura direta e plantio de mudas produzidas em tubetes, na implantação de povoamentos de Pinus taeda, L. Curitiba: UFPR, 1993. 149f. Tese Doutorado

PARVIAINEN, J. V. Containerized forest tree seedling prodution in Finland and the other nordic countries, In: SIMPÓSIO INTERNACIONAL SOBRE MÉTODOS DE PRODUÇÃO E CONTROLE DE QUALIDADE DE SEMENTES E MUDAS FLORESTAIS, 1984, Curitiba. Métodos de Produção e Controle de Qualidade de Sementes e mudas Florestais. Curitiba: UFPR/FUPEF, 1984. p.403-417.

PARVIAINEN, J. V. Future trends for containerized tree seedlings production: a literature review. Silva Fennica, Helsinki, v.24, n.1, p.93-103, 1990.

PARVIAINEN, J. V.; ANTOLA, J. The root system morphology and stand development of different types of pine nursery stock. Folia Forestalia, Helsink, v.671, p.1-29, 1986.

PARVIAINEN, J. V.; TERVO, L. A new approach for prodution of containerized coniferous seedlings using peat sheets coupled with root pruning. Forestry Supplement, Oxford, v.62, p.87-94, 1989.

RICHTER. J. Das Umsetzen von Douglasien in Kulturstadium. Allg. Forst.-u. Jagdztg., Frankfurt, v.142, p.63-69, 1971

SCHMIDT-VOGT, H. Wachstum und Qualitaet von Forstpflanzen. 2. ed. Munique: Bayerischer Landwirtschaftverlag. 1966. $210 \mathrm{p}$.

SCHMIDT-VOGT, H.; GÜRTH, P. Eigenschaften von Forstpflanzen und Kulturerfolg - I. Mitteilung: Auspflanzungsversuche mit fichten-und kieferpflanzen verschiedener grössen und Durchmesser. Allg Forst-u. Jagdztg., Frankfurt, v. 140, n.6 p.132-142, 1969.

SCHMIDT-VOGT, H. Morpho-physiological quality of forest tree seedlings: the present international status of research. In: SIMPÓSIO INTERNACIONAL SOBRE MÉTODOS DE PRODUÇÃO E CONTROLE DE QUALIDADE DE SEMENTES E MUDAS FLORESTAIS. Curitiba, 1984, Anais...Curitiba: UFPR/FUPEF, 1984. p.366-378.

SJOBERG, N. E. The styroblock container system. In: NORTH AMERICAN CONTAINERIZED FOREST TREE SEEDLING SYMPOSIUM, 1974: Denver, Col. Proceedings... Washington, D. C. U. S. Government Printing Office, 1974. p.217-228. (Great Plains Agric. Council Publication, 68).

SOUTH, D. B. ZWOLINSKI, J. B. DONALD, D. G. M. Interations among seedling diameter grade, weed control and soil cultivation for Pinus radiata in South Africa. Can. J. Res., Ottawa, v.23, p.20782082, 1993.

TINUS, R. W.; McDONALD, S. E. How to grow tree seedlings in containers in greenhouses. Gen. Tech. Rep. RM. USDA. For. Serv., Fort Collins, Colorado, n. 60, p. 1-256, 1979. 